\section{Dentin matrix protein 1 and dentin sialophosphoprotein in human sound and carious teeth: an immunohistochemical and colorimetric assay}

\author{
D. Martini, ${ }^{1}$ A. Trirè, ${ }^{1}$ L. Breschi, ${ }^{2,3}$ \\ A. Mazzoni, ${ }^{2,3}$ G. Orsini, ${ }^{4}$ \\ G. Teti, ${ }^{1}$ M. Falconi, ${ }^{1}$ A. Ruggeri Jr. ${ }^{1}$ \\ 'Department of Biomedical \\ and Neuromotor Sciences, \\ University of Bologna \\ 2Department of Medical Sciences, Unit \\ of Dental Sciences and Biomaterials, \\ University of Trieste \\ 3IGM, Unit of Bologna, CNR-IOR, Bologna \\ ${ }^{4}$ Department of Clinical Sciences and \\ Stomatology, Polytechnic University \\ of Marche, Ancona, Italy
}

\section{Abstract}

Dentin matrix protein 1 (DMP1) and dentin sialophosphoprotein (DSPP) are extracellular matrix proteins produced by odontoblasts involved in the dentin mineralization. The aim this study was to compare the distribution of DMP1 and DSPP in human sound dentin vs human sclerotic dentin. Sixteen sound and sixteen carious human molars were selected, fixed in paraformaldehyde and processed for immunohistochemical detection of DMP1 and DSPP by means of light microscopy, transmission electron microscopy (TEM) and high-resolution field emission in-lens scanning electron microscopy (FEI-SEM). Specimens were submitted to a pre-embedding or a postembedding immunolabeling technique using primary antibodies anti DMP1 and anti-DSPP and gold-conjugated secondary antibodies. Other samples were processed for the detection of DMP1 and DSPP levels. Dentin from these samples was mechanically fractured to powder, then a protein extraction and a protein level detection assay were performed. DMP1 and DSPP were more abundant in carious than in sound samples. Immunohistochemical analyses in sclerotic dentin disclosed a high expression of DMP1 and DSPP inside the tubules, suggesting an active biomineralization of dentin by odontoblasts. Furthermore, the detection of small amounts of these proteins inside the tubules far from the carious lesion, as shown in the present study, is consistent with the hypothesis of a preventive defense of all dentin after a noxious stimulus has undermined the tooth.

\section{Introduction}

Dentin matrix protein 1 (DMP1) and dentin sialophosphoprotein (DSPP) are two non-collagenous extracellular matrix proteins belonging to the SIBLING (Small Integrin Binding LIgand N-linked Glycoprotein) family, a group of acidic and highly phosphorylated proteins involved as key factors in the mineralization process of dentin and bone. ${ }^{1-4}$ In vitro and in vivo studies supported the hypothesis that DMP1 is involved in the initial phases of mineralized dentin formation. Indeed, this protein has been shown to act as a hydroxyapatite nucleator in vitro. ${ }^{5,6}$ In particular, dmp1-null mice showed defects in odontogenesis and in dentin mineralization, including hypomineralization, partial failure in predentin maturation into dentin, and alterations in the tubular system formation. ${ }^{7,8}$ Previous studies also revealed the contribution of DMP1 mutations to human tooth development, even if the related mechanisms are still to be elucidated. ${ }^{9}$ Additionally, DMP1 in both bone and dentin is proteolytically cleaved into a N-terminal (Nter) (37 kDa) and a C-terminal (C-ter) (57 $\mathrm{kDa}$ ) fragments, which are released in the extracellular matrix as full-length DMP1 and its cleavage products. ${ }^{10,11}$ These fragments seem to be involved in the biomineralization process of dentin, ${ }^{9,12}$ but their specific roles have not been fully clarified. ${ }^{13-16}$ The contribution of $d m p 1$ mutations to human tooth development was also hypothesized, but the related mechanisms are unclear. ${ }^{8}$ As regards DSPP, knockout experiments revealed its importance in dentin mineralization; $d s p p$-deficient mice showed hypomineralization of predentin and defects in the mineralization process of the dentin matrix:5,17,18 indeed a relationship between human dentinogenesis imperfecta and $d s p p$ mutations has been well established. ${ }^{19-21}$ Similarly to DMP1, DSPP is proteolytically cleaved in two products, dentin sialoprotein (DSP) and dentin phosphoprotein (DPP) ${ }^{22-24}$ Basing on previous experimental studies, ${ }^{11,25,26}$ Prasad et al. recently formulated the hypothesis that DSPP is cleaved into odontoblasts, and subsequently DSP is released within the predentin, while DPP is released at the mineralization front and retained within the mineralized dentin. ${ }^{16}$ Therefore, DSP and DPP may play distinct roles in the biomineralization process (i.e., mineralization by living cells). ${ }^{14-15,27}$

Based on these findings, Qin hypothesized that both full-length DMP1 and DSPP are inactive precursors and the proteolytic processing of these proteins results in the formation of their active fragments, which play significant roles in the biomineralization of dentin. ${ }^{2}$ All these data lead to hypothesize a synergistic
Correspondence: Prof. Alessandra Ruggeri Jr., Department of Biomedical and Neuromotor Sciences - DIBINEM, University of Bologna, via Irnerio 48, 40126 Bologna, Italy.

Tel. +39.051 .2091511 - Fax: +39.051 .251735 .

E-mail: alessandra.ruggeri@unibo.it

Key words: sclerotic dentin, dentin matrix protein, dentin sialophosphoprotein, immunohistochemistry.

Contributions: DM, study conception and design, experiments performing; AR Jr, study conception and design, data analysis and interpretation, article drafting and critical revision and final version approval; AT, experiments performing, data analysis and interpretation; LB, AM, G0, data analysis and interpretation; GT, data analysis and interpretation, article drafting and critical revision; $\mathrm{MF}$, article final version approval.

Received for publication: 25 July 2013. Accepted for publication: 20 September 2013.

This work is licensed under a Creative Commons Attribution NonCommercial 3.0 License (CC BYNC 3.0).

(C) Copyright D. Martini et al., 2013

Licensee PAGEPress, Italy

European Journal of Histochemistry 2013; 57:e32 doi:10.4081/ejh.2013.e32

function of DMP1 and DSPP and indicate that the active fragments obtained from the cleavage of DSPP may interact with those of DMP1 within the dentin matrix. ${ }^{16}$ In a previous study $\mathrm{DMP}^{28}$ we analyzed the DMP1 distribution in human sound dentin by an immunofluorescence and high-resolution immunogold labeling techniques. Many studies have also described the quantification and distribution of DSPP protein in sound dentin, too. As concerning their distributions in human carious dentin has not been elucidated yet.

Hence, the aim of the present study was to investigate the presence and distribution of DMP1 and DSPP by means of a correlative immunohistochemical and colorimetric assay in human sound and sclerotic dentin. The null hypothesis tested was that no difference could be found in DMP1 and DSPP expression between sound $v s$ sclerotic dentin.

\section{Materials and Methods}

Sixteen sound and sixteen carious human molars were selected for orthodontic reasons (selection criteria: carious lesions closer to 1 $\mathrm{mm}$ or less from the pulp chamber were not included in the study) after informed consent form was obtained by patients under an exper- 
imental protocol approved by the Ethics Committee of the University of Bologna (Italy). After extraction, roots were removed with a low speed diamond saw (Sawing and Grinding System, Remet, Bologna, Italy) under water cooling, while the corresponding crowns were randomly and equally assigned to four different groups ( $\mathrm{N}=4$ sound teeth and $\mathrm{N}=4$ carious teeth/group).

\section{Group 1}

\section{Light microscopy analysis}

Specimens were rinsed for $10 \mathrm{~min}$ each in $0.1 \mathrm{M}$ cacodylate buffer $(\mathrm{pH}=7.4)$ at $4^{\circ} \mathrm{C}$, fixed overnight in $4 \%$ paraformaldehyde in $0.1 \mathrm{M}$ sodium cacodylate buffer at $4^{\circ} \mathrm{C}$, rinsed in sodium cacodylate buffer for $10 \mathrm{~min}$ at room temperature (RT), then washed in distilled water for $30 \mathrm{~min}$ at RT. Fixed specimens were dehydrated in an ascending ethanol series $\left(50^{\circ}, 70^{\circ}, 80^{\circ}, 90^{\circ}, 95^{\circ}, 100^{\circ}\right.$, renewing each solution twice for $60 \mathrm{~min}$ at RT), defatted in xylol for $120 \mathrm{~min}$ at RT, and embedded in a methylmethacrylate-based resin system (Technovit 9100 New, Heraeus Kulzer, Hanau, Germany). After resin polymerization, each specimen was longitudinally sectioned, glued to plastic slides using a methylmethacrylatebased glue (Technovit 7210 VLC, Heraeus Kulzer, Hanau, Germany) then grinded and polished with wet-paper (up to 1200 grit) to obtain 100-150 $\mu$ m-thick sections (Sawing and Grinding System, Remet). Sections were then processed for immunohistochemical analysis. ${ }^{29}$ Briefly, sections were rinsed for $5 \mathrm{~min}$ in distilled water, pre-treated with $0.5 \mathrm{M}$ ethylenediaminetetraacetic acid (EDTA) for $30 \mathrm{~min}$ at RT, rinsed for $10 \mathrm{~min}$ in distilled water, rinsed in $1 \mathrm{X}$ phosphate buffered saline (PBS, GIBCO, Life Technologies, Carlsbad, CA, USA; $\mathrm{pH}$ 7.6) twice for $10 \mathrm{~min}$ at RT, incubated for 10 min in the peroxidase blocking solution included in the horseradish peroxidase (HRP)based detection system selected for secondary labeling (UltraVision Quanto Detection System HRP DAB, Thermo Fisher Scientific, Fremont, CA, USA). Specimens were then rinsed in PBS three times for $10 \mathrm{~min}$ at RT, pre-incubated with normal goat serum (NGS) in PBS for 30 min at RT, then incubated overnight at $4{ }^{\circ} \mathrm{C}$ either with a mouse monoclonal antibody anti-DMP1 at 1:50 (Santa Cruz Biotechnology, Santa Cruz, CA, USA) or with a rabbit polyclonal antibody anti-DSPP (Sigma Aldrich, St. Louis, M0, USA). Specimens were rinsed in PBS for 10 min at RT, then processed for antibody detection using a polymeric labeling with the horseradish peroxidase (HRP)based detection system (UltraVision Quanto Detection System HRP DAB, Thermo Fisher Scientific). Specimens were then rinsed three times in distilled water at RT, air dried, and observed under a light microscopy (BX41, Olympus Optical Co. Europa GmbH, Hamburg, Germany). Images were taken at 10x and 100x magnification by two independent blind observers. As negative control, a section from each tooth was processed without the primary antibody.

\section{Group 2}

Field emission in-lens scanning electron microscopy analysis

Crown specimens were rinsed in $0.1 \mathrm{M}$ cacodylate buffer $(\mathrm{pH}=7.4)$, fixed in $4 \%$ paraformaldehyde buffered with $0.1 \mathrm{M}$ sodium cacodylate and processed for an immunolabeling procedure in accordance with previous immunohistochemical studies. ${ }^{29,30,31}$ In brief, specimens were longitudinally cut using a low speed diamond saw (Sawing and Grinding System, Remet) under continuous water-cooling to obtain $1 \mathrm{~mm}$-thick dentin sections. Samples were then polished by increasing grid $\mathrm{SiC}$ paper under constant deionized water irrigation and ultrasonically cleaned in $0.05 \mathrm{M}$ Tris $\mathrm{HCl}$ (Trizma Hydrocloride, Sigma Aldrich) buffer solution (TBS) at pH 7.6. Specimens were then pre-incubated in NGS in TBS and then incubated overnight at $4^{\circ} \mathrm{C}$ with the same primary antibodies used in Group 1 (i.e., light microscopy analysis), thus either with an antiDMP1 or an anti-DSPP. Specimens were then rinsed with TBS $0.05 \mathrm{M}$ at pH 7.6 and TBS 0.02 $\mathrm{M}$ at $\mathrm{pH} 8.2$, then DMP1 detection was performed incubating the sections with a goat anti-mouse secondary antibody (British BioCell International, Cardiff, UK), while DSPP detection was achieved with a mouse anti-rabbit secondary antibody (British BioCell International). Both secondary antibodies were conjugated with gold nanoparticles of 15 $\mathrm{nm}$ in diameter for electron microscopy visualization and diluted in $0.02 \mathrm{M}$ TBS at $\mathrm{pH} 8.2$ (0.02 M Tris $\mathrm{HCl}$ buffered at $\mathrm{pH} 8.2$ with $0.15 \mathrm{M}$ $\mathrm{NaCl}$ and $0.1 \% \mathrm{BSA})$. Specimens were then rinsed in TBS $0.02 \mathrm{M}$ at $\mathrm{pH} 8.2$, washed in deionized water, dehydrated in ascending ethanol series, air dried from hexamethyldisilazane (HMDS) and coated with carbon graphite. Observations were performed under a Field Emission In-lens Scanning Electron Microscope (JEOL JSM 890, JEOL, Tokyo, Japan) at $10 \mathrm{KV}$ accelerating voltage and $1 \times 10^{-11}$ Amp probe current. Final images were obtained by mixing both back scattered (BSI) and secondary electron (SEI) signals.
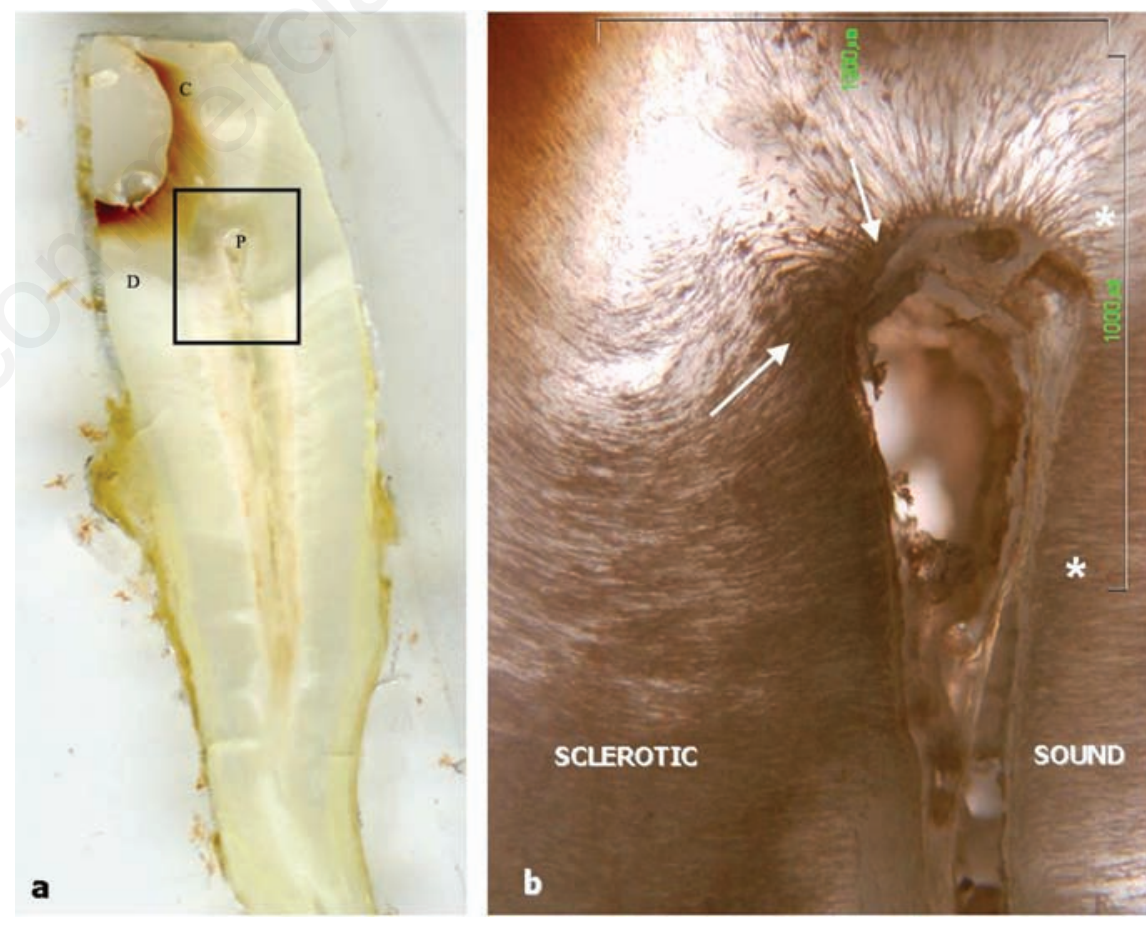

Figure 1. Section of a tooth sample at a low magnification within carious lesion. a) Longitudinal section of tooth with a carious process from enamel to deep dentin. Black box point out in particular sclerotic dentin and sound dentin area on the opposite site. D, dentin; P, pulp; C, carious lesion; b) Detail at a high magnification of black box area. The figure show an immunolabeling of one of the two proteins (DMP1) on sclerotic dentin (arrows) vs sound dentin (asterisks). In sclerotic dentin layer is evident an higher staining. Scale bars: $1000 \mu \mathrm{m}$. Following Figures 2 and 3 show show both proteins, DMP1 and DSPP, respectively, demonstrating demonstrating an increasing of staining of the immunolabeling on sclerotic dentin. 
As negative control, a section from each tooth was processed without the primary antibody.

\section{Group 3}

Transmission electron microscopy analysis

After fixation, disks were washed for $1 \mathrm{~h}$ in $0.1 \mathrm{M}$ cacodylate buffer, $\mathrm{pH} 7.4$, and decalcified using $4.13 \%$ EDTA for three months at $4^{\circ} \mathrm{C}$. Specimens were then extensively washed with $0.1 \mathrm{M}$ sodium cacodylate buffer, dehydrated in graded concentrations of ethanol and embedded in LR White resin (London Resin, Berkshire, UK). Semi-thin sections $(1 \mu \mathrm{m})$ were cut with glass knives on a Reichert Jung Ultracut E Ultramicrotome and stained with toluidine blue. Selected areas of the sample were trimmed for ultra thin sectioning (80 $\mathrm{nm}$ ) using a diamond knife. Sections were mounted on Formvar carbon-coated nickel grids. Grid-mounted tissue sections were processed for immunocytochemical labelling, with anti-DMP1 ${ }^{28}$ and anti-DSPP, as previously reported. After labeling, grids were stained with $4 \%$ uranyl acetate and lead citrate for examination in a TEM (CM-10; Philips, Eindhoven, The Netherlands) operating at 70 $\mathrm{kV}$. Transmission electron microscopy analysis (TEM) images were outfitted with a MegaView III Digital Camera (FEI) operating under the Analysis Imaging System $\mathrm{GmbH}$ (Munster, Germany). Controls consisted of sections incubated i) with secondary antibody only; ii) substituting the primary antisera with nonimmune serum.

\section{Group 4}

\section{Colorimetric assay}

Roots were cut off from the selected teeth using the low speed diamond saw and enamel was completely removed using a grinding machine. Dental pulp remnants and predentin, were removed thus obtaining dentin specimens that were processed for a protein detection assay. Specimens were mechanically fractured with a steel mortar/pestle (Reimiller, Reggio Emilia, Italy), in accordance to Mazzoni et al. ${ }^{32}$ in order to obtain a fine dentin powder from both sound and carious teeth. The dentin powder was then decalcified with $1 \%$ phosphoric acid for $10 \mathrm{~min}$ at $4^{\circ} \mathrm{C}$ and processed for protein extraction. Dentin powder was buffered at $\mathrm{pH}$ 7.0 with $4 \mathrm{M} \mathrm{NaOH}$, centrifuged at 14,000 rpm for $20 \mathrm{~min}$ at $4^{\circ} \mathrm{C}$ (Eppendorf MiniSpin Plus, Hamburg, Germany), supernatant was discarded, then specimens were re-suspended overnight at $4^{\circ} \mathrm{C}$ in the extraction buffer provided with the quantification kit (Quantisir, Epigentek, Brooklyn, NY, USA), centrifuged at $14,000 \mathrm{rpm}$ for $20 \mathrm{~min}$ at $4^{\circ} \mathrm{C}$ then the supernatant was collected and re-centrifuged. Supernatant was then ultrafiltrated (Vivaspin,
Sartorius Stedim Biotech, Aubagne, France) and centrifuged at $14,000 \mathrm{rpm}$ for $30 \mathrm{~min}$ at $4^{\circ} \mathrm{C}$, to obtain the protein extract. The overall protein level in the protein extract was measured with a Bradford assay (Nanodrop 2000c, Thermo Fisher Scientific). DMP1 and DSPP level detec-
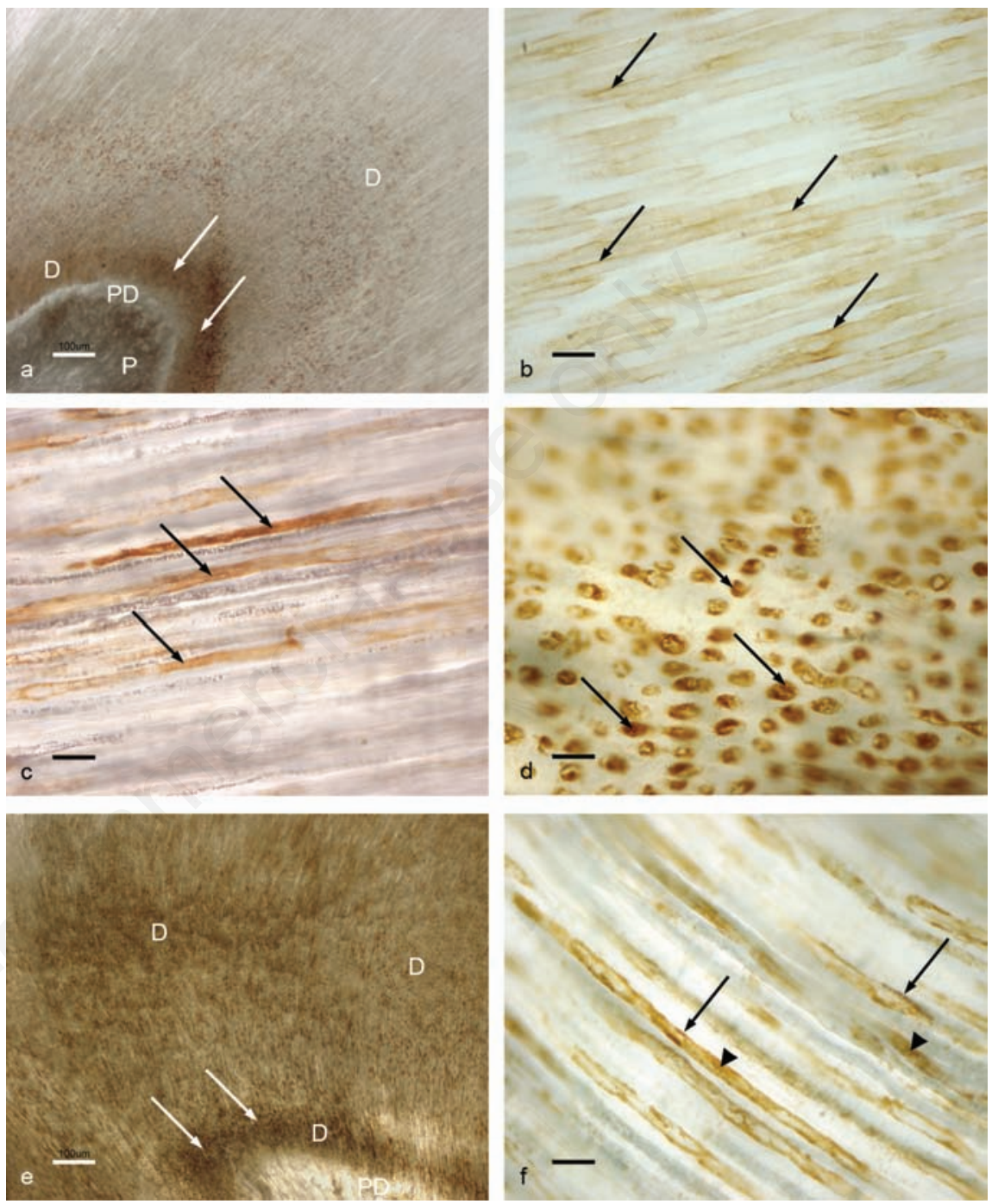

Figure 2. Sections of sound and carious samples immunolabeled for the DMP1 detection (light microscopy). D, dentin; P, pulp; PD, predentin. a) Longitudinal section of a sound sample; in close proximity to predentin, a very intense $D A B$ staining of dentin can be observed (arrows). Labeling of dentin shows a decreasing distribution moving towards enamel; scale bar: $100 \mathrm{~m}$. b) Detail of sound dentin from a sound sample (longitudinal section); a mild labeling can be observed in peritubular dentin (arrows); scale bar: $17 \mathrm{~m}$. c) Detail of sclerotic dentin from a carious sample (longitudinal section); an intense immunostaining of peritubular and intratubular dentin can be observed (arrows); scale bar: $17 \mathrm{~m}$. d) Detail of sclerotic dentin from a carious sample (transversal section); the immunostaining can be clearly detected inside several tubules (arrows); scale bar: $17 \mathrm{~m}$. e) Longitudinal section of a carious sample; the image shows an area of non-affected dentin; the labeling pattern is similar to the one observed in sound dentin (Figure 1a), but the immunostaining here appears more intense; observe the very intense immunolabeling of dentin in proximity to predentin (arrows); scale bar: $100 \mathrm{~m}$. $\mathrm{f}$ ) Detail of non-affected dentin from a carious sample (longitudinal section); labeling in peritubular dentin (arrows) is more intense than that observed in sound dentin (Figure 1b); a moderate immunostaining can be observed into some of the tubules (arrowheads); scale bar: $17 \mathrm{~m}$. 
the protein capture buffer were used for blank. Strip wells were incubated for $90 \mathrm{~min}$ at $37^{\circ} \mathrm{C}$, then $150 \mu \mathrm{L}$ of the blocking buffer were added to each strip well and incubated for $30 \mathrm{~min}$ at $37^{\circ} \mathrm{C}$. Wells were then washed three times with $1 \mathrm{X}$ wash buffer, incubated for $60 \mathrm{~min}$ at RT on an orbital shaker $(50 \mathrm{rpm})$ with the same primary antibodies used in Group 1 and 2, thus either with an anti-DMP1 or an anti-DSPP, diluted to $1 \mu \mathrm{g} / \mu \mathrm{L}$ in the antibody buffer. The antibody buffer alone served as a negative control. Wells were then washed four times with $1 \mathrm{X}$ wash buffer and incubated with the detection antibodies for 30 min at RT: DMP1 detection was performed incubating the sections with a goat horseradish peroxidase-conjugated antimouse secondary antibody (Bethyl Laboratories Inc., Montgomery, TX, USA), while DSPP detection was achieved with a horseradish peroxidase-conjugated anti-rabbit secondary antibody (Sigma Aldrich). Both detection antibodies were diluted 1:1000 in the antibody buffer. Wells were then washed five times with $1 \mathrm{X}$ wash buffer and incubated in the developing solution for 3 min at RT away from light, constantly monitoring color development. The stop solution was then added to the developing solution in the wells and the absorbance values were read at $450 \mathrm{~nm}$ on a microplate reader (LT 4000, Labtech International Ltd, Lewes, United Kingdom). Statistical analysis was performed with a Student $t$-test.

\section{Results}

\section{Group 1}

\section{Light microscopy analysis}

Section of a tooth sample at a low magnification within carious lesion (Figure 1). Sound dentin specimens showed an intense ringshaped labeling of DMP1 at the predentin layer up to $600-700 \mu \mathrm{m}$ from the mineralization front (at the predentin-dentin border) gradually decreasing towards the dentin-enamel junction (Figure 2a). At higher magnification, mild DMP1 labeling was mainly localized in peritubular dentin (Figure 2b). In carious specimens, the sclerotic dentin layer surrounding the carious lesion showed an intense DMP1 labeling, involving both peritubular and intratubular dentin (Figure 2 c,d). Indeed, immunostaining was higher than in sound teeth. Additionally, increased DMP1 labeling compared to sound teeth could be observed in the sound dentin areas of the carious teeth (i.e., non affected dentin areas, 1000 - $1500 \mu \mathrm{m}$ far from the carious lesion) (Figure 2e). In these areas, intense labeling at the peritubular level and minor labeling in intratubular dentin could be detected (Figure $2 \mathrm{f}$ ).
DSPP labeling was intense in sound specimens, particularly at the predentin-dentin front of mineralization, decreasing towards the dentin-enamel junction, up to $400-500 \mu \mathrm{m}$ distance from the predentin-dentin border (Figure 3a). Similarly to DMP1, high magnification images confirmed a mild labeling mainly localized in the peritubular dentin (Figure 3 b). In carious specimens, the sclerotic dentin
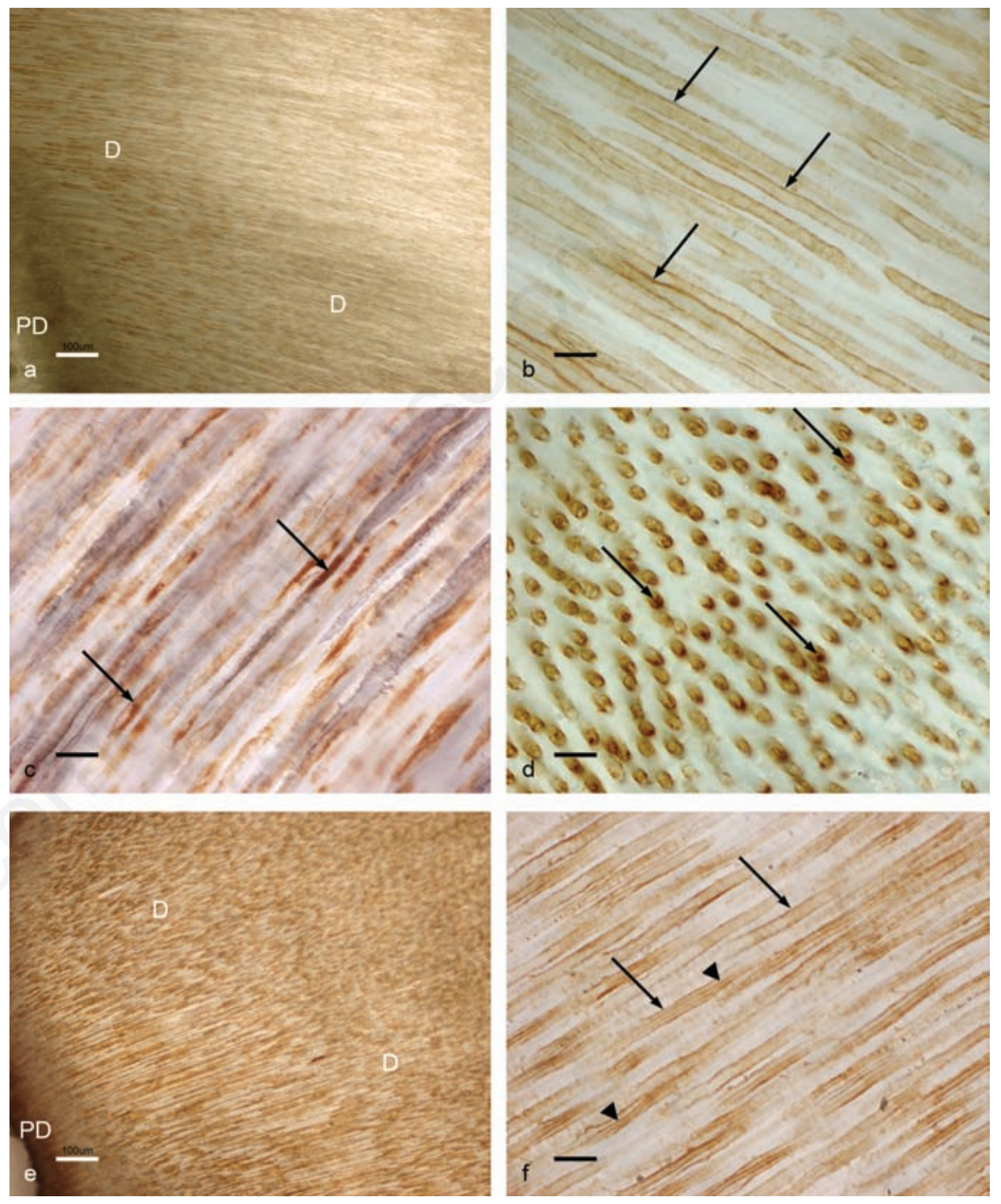

Figure 3. Sections of sound and carious samples immunolabeled for DSPP detection (light microscopy); D, dentin; PD, predentin. a) Longitudinal section of a sound sample; a moderate $\mathrm{DAB}$ staining can be observed; a decreasing labeling pattern can be seen moving towards enamel; scale bar: $100 \mathrm{~m}$. b) Detail of sound dentin from a sound sample (longitudinal section); immunostaining is mildly distributed in peritubular dentin (arrows); scale bar: $17 \mathrm{~m}$. c) Detail of sclerotic dentin from a carious sample (longitudinal section); an intense immunolabeling of intratubular dentin can be observed in some of the tubules (arrows); scale bar: $17 \mathrm{~m}$. d) Detail of sclerotic dentin from a carious sample (transversal section); immunolabeling can be detected inside the tubules (arrows); scale bar: $17 \mathrm{~m}$. e) Longitudinal section of a carious sample; the image shows an area of non-affected dentin; the labeling pattern is similar to that observed in sound dentin (Figure 2a) but markedly stronger; scale bar: $100 \mathrm{~m}$. f) Detail of non-affected dentin from a carious sample (longitudinal section); a moderate labeling can be detected both in peritubular dentin (arrows) and along the walls of odontoblast processes (arrowheads); scale bar: $17 \mathrm{~m}$. 
tubules (Figure 3e). A moderate labeling could be also detected along the walls of the odontoblast processes (Figure 3f).

No immunoreactivity for DMP1 or DSPP was detected within the enamel layer and in all control sections (data not shown).

\section{Group 2}

Field emission in-lens scanning electron microscopy analysis

Ultrastructural investigations confirmed the light microscopy findings, i.e., positive immunolabeling was observed for DMP1 and DSPP in both sound and carious dentin. As regards DMP1, a weak immunolabeling was observed in sound dentin with gold nanoparticles distributed along the peritubular dentin (data not shown). In carious dentin specimens, sclerotic tubules are totally filled (Figure 4a) and showed a very intense immunolabeling of both peritubular and intratubular dentin even along the walls of the odontoblastic processes (Figure 4b). In nonaffected dentin areas, 1000 - $1500 \mu \mathrm{m}$ far from the carious lesion, a positive but minor labeling anti-DMP1 intratubular dentin could be detected in the dentinal tubules (Figure $4 \mathrm{c}, \mathrm{d}$ ).

DSPP showed a weak immunolabeling in sound dentin specimens, with few nanogold particles distributed over peritubular dentin (data not shown). Carious dentin specimens showed DSPP labeling in sclerotic dentin (Figure 5a) showing many nanogold particles in intratubular and peritubular dentin of the odontoblastic processes (Figure 5b). At 1000 $1500 \mu \mathrm{m}$ distance from the carious lesion (Figure $5 \mathrm{c}, \mathrm{d}$ ) some of the non-caries affected tubules revealed labeling of the intratubular and peritubular dentin (Figure 5d).

No labeling for DMP1 or DSPP was detected in negative controls (data not shown).

\section{Group 3}

Transmission electron microscopy analysis

TEM micrographs obtained from demineralized sections, confirm the intensive labeling of proteins DMP1 and DSPP in carious dentin (Figure 6 a-d). In particular, in sclerotic dentin sections tubules in both DMP1 and DSPP micrographs appear filled, as revealed in the field emission in-lens scanning electron microscopy (FEISEM) findings, and rich of gold particles (Figure 6 a,c); while in non affected dentin DMP1 and DSPP sections (Figure 6 b,d) labeling is less intensive.

\section{Group 4}

\section{Colorimetric assay}

Mean values of DMP1 and DSPP absorbance in protein extracts obtained from carious and sound samples are shown in Table 1. DMP1 levels in carious dentin were $37 \%$ higher than in sound dentin. DSPP levels in carious dentin were $73 \%$ higher than in sound dentin. Statistical analysis for both DMP1 and DSPP showed significant differences between sound and carious dentin $(\mathrm{P}<0.05)$ (Table 1$)$. In DMP1 and DSPP levels both inactive precursors and active fragments are included.

\section{Discussion}

The antibodies used in the present study bind to the inactive precursors (DMP1, DSPP) as well as to their related active products, therefore the distribution and levels of DMP1 and DSPP as detected in the immunohistochemical and quantitative analyses always refer to both precursors and products.

The significant increase of DMP1 and DSPP levels detected in carious dentin vs sound dentin, as shown by both immunohistochemical and quantitative analyses allows the rejec-
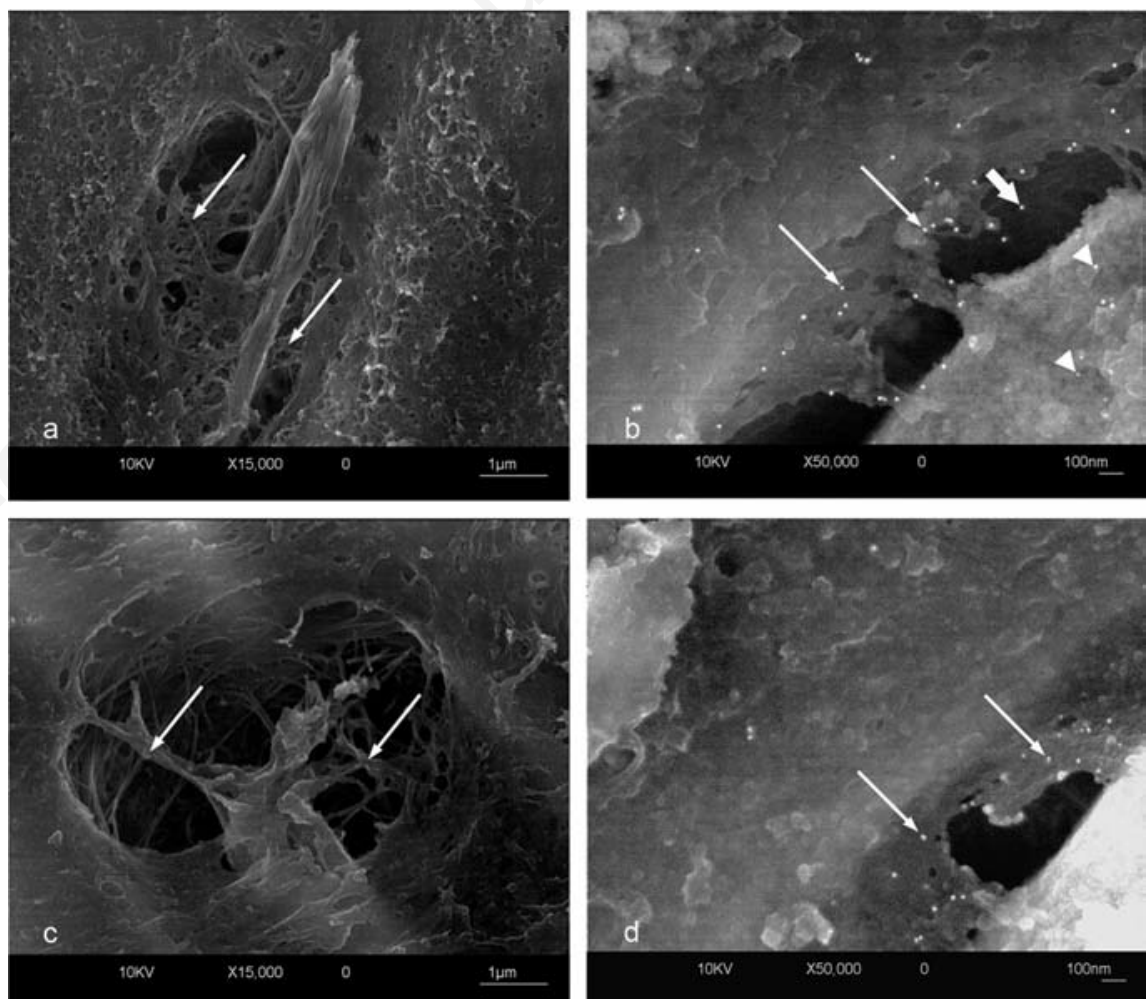

Figure 4. Sections of dentin from a carious sample immunolabeled for dentin matrix protein 1 detection (FEISEM). a) Transversal section of a tubule in sclerotic dentin; observe the trabeculae of intratubular dentin almost completely filling the tubule lumen (arrows); scale bar: $1 \mathrm{~m}$. b) Transversal section of a tubule in sclerotic dentin at a higher magnification; gold particles can be detected in both intratubular and peritubular dentin (arrows) and along the walls of the odontoblast process (arrowheads); scale bar: $100 \mathrm{~nm}$. c) Transversal section of a tubule in non-affected dentin; note the trabeculae of intratubular dentin partially occluding the tubule lumen (arrows); scale bar: $1 \mathrm{~m}$. d) Transversal section of a tubule in non-affected dentin at a higher magnification; a mild gold immunolabeling can be detected in intratubular dentin (arrows); scale bar: $100 \mathrm{~nm}$. 

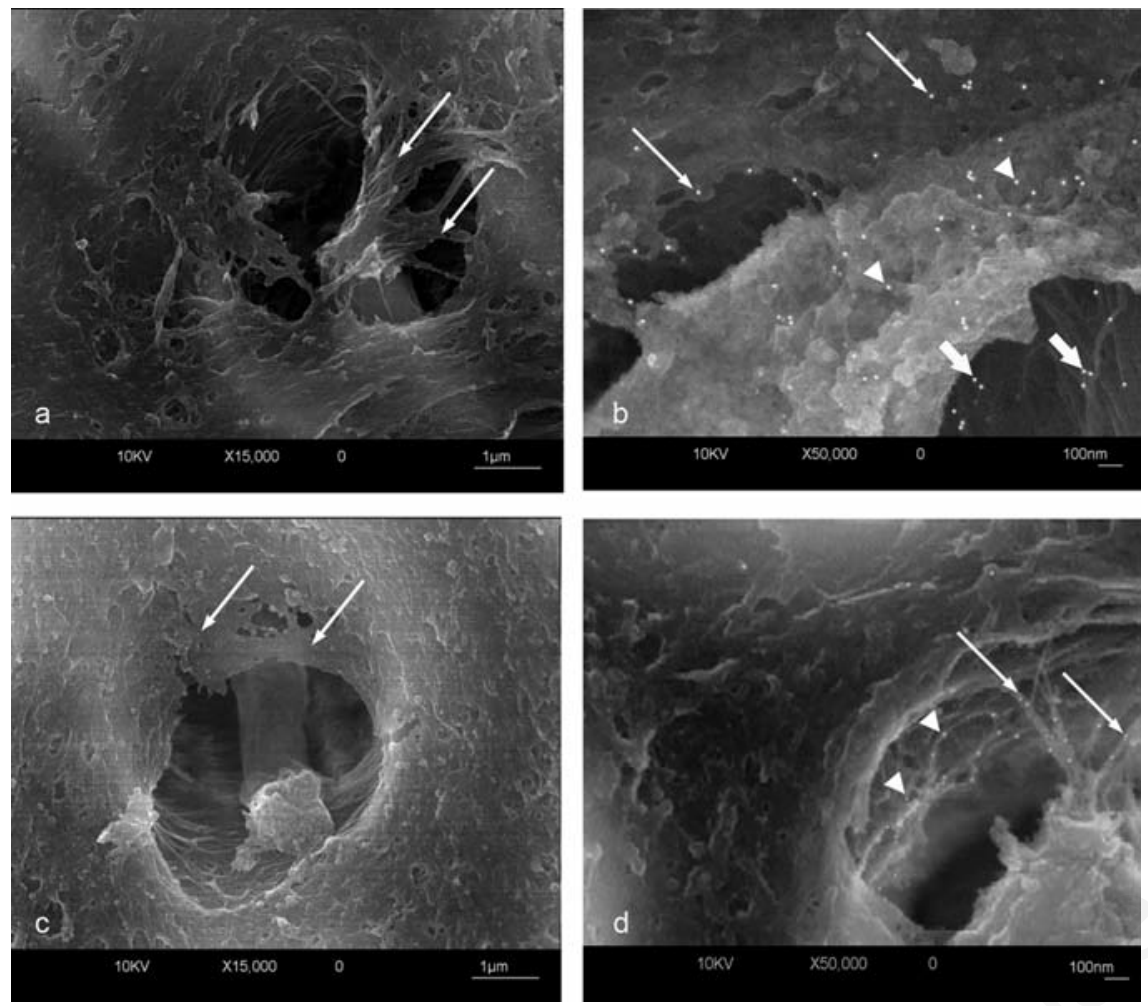

Figure 5. Sections of dentin from a carious sample immunolabeled for dentin sialophosphoprotein detection (FEISEM). a) Transversal section of a tubule in sclerotic dentin; the trabeculae of intratubular dentin fill the tubule lumen (arrows); scale bar: $1 \mathrm{~m}$. b) Transversal section of a tubule in sclerotic dentin at a higher magnification; immunolabeling can be detected in intratubular (long arrows) and peritubular (short arrows) dentin; numerous gold particles are distributed along the walls of the odontoblast process (arrowheads); scale bar: $100 \mathrm{~nm}$. c) Transversal section of a tubule in non-affected dentin; note the intratubular dentin partially occluding the tubule lumen (arrows); scale bar: $1 \mathrm{~m}$. d) Transversal section of a tubule in nonaffected dentin at a higher magnification; minor gold particles can be observed in peritubular (arrowheads) and intratubular (arrows) dentin; scale bar: $100 \mathrm{~nm}$.
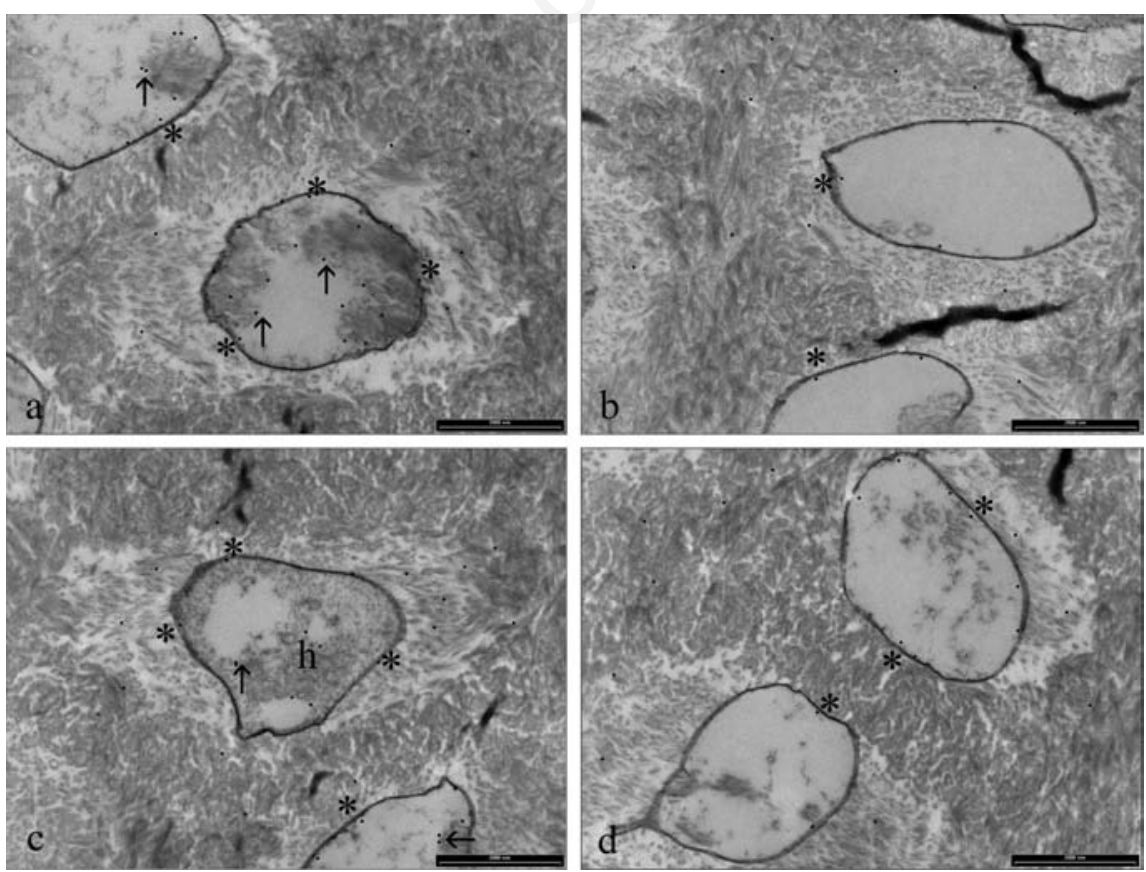

Figure 6. Sections of dentin from sclerotic and non affected dentin samples immunolabeled for both DMP1 and DSPP proteins detection (TEM). a) Section immunolabeled for DMP1 sclerotic dentin; dentin tubule lumen appear filled and labeling is intensive in peritubular space $(*)$ and intratubular space (arrows) as to confirm results obtained in both light and FEISEM microscopy; scale bar: 2000 nm. b) Section immunolabeled for DMP1 non-affected dentin; banded collagen fibrils along the cut dentin surface in intertubular space are partially raveled; a few gold particles are present and localized in peritubulare space $\left(^{*}\right)$; scale bar: $2000 \mathrm{~nm}$. c) Section immunolabeled for DSPP sclerotic dentin; numerous gold particles are distributed along the walls and inside the tubles (arrows, *); scale bar: $2000 \mathrm{~nm}$. d) Section immunolabeled for DSPP nonaffected dentin; note the tubule lumen is not occluded or partially occluded in sclerotic section $\left(^{*}\right)$; scale bar: $2000 \mathrm{~nm}$. 
complete occlusion of tubule lumens. ${ }^{33,34}$ In response, sclerotic dentin creates a barrier at the bottom of the carious lesion, to prevent caries progression towards the pulp..$^{5,35}$ Several studies have been carried out to clarify the mechanisms underlying sclerotic dentin formation in response to carious stimuli, but the results are controversial and the process leading to dentin sclerosis as well as its proteins mediated regulation is still matter of investigation. Although some authors suggested that intratubular dentin apposition may result from re-precipitation of dissolved dentin, ${ }^{36-38}$ the vast majority of authors supports the hypothesis that sclerotic dentin originates from an active secretion of different organic proteins by odontoblast processes, regulating the formation and mineralization of intratubular dentin. ${ }^{33,35,39-43}$

DMP1 and DSPP have been shown to actively take part to the dentin biomineralization, thus their presence in the intratubular sclerotic dentin and on the odontoblast processes in carious samples shown in the present study, supports the hypothesis of an active biomineralization process of the dentin matrix carried out by the odontoblasts underlying sclerotic dentin formation. Furthermore, the detection of small amounts of intratubular dentin immunoreactive to anti-DMP1 and anti-DSPP in the tubules at a certain distance from the carious lesion (the non-affected dentin) might be considered as a preparatory response of non-affected dentin to the carious attack.

An early response by the odontoblasts to the carious stimuli after the lesion reaches the enamel-dentin junction or even when it starts eroding enamel has been reported. ${ }^{44,45}$ Thus, the observed response might fasten the occlusion of tubules if the carious lesion should extend to different areas of the tooth. The labeling for both DSPP and DMP1 in nonaffected dentin was more intense than the labeling of the corresponding areas in sound teeth. This result is consistent with the hypothesis of a preventive defense of nonaffected dentin against a potential damage, after a noxious stimulus has undermined the tooth. The permeability of dentin ${ }^{46,47}$ consequent flow of molecules between dentin and pulp can contribute to explain the presence of this hypothesized diffuse response to caries even in non-affected dentin. The lack of labeling for both DMP1 and DSPP at a certain distance from the predentin-dentin border is consistent with the hypothesis that odontoblast processes do not reach the dentin-enamel junction. In fact, though there is no general agreement on the length of the odontoblast processes, many studies indicate that these processes do not extend throughout dentin, being shorter than the dentin tubules that host them. ${ }^{47,48}$
In this perspective, investigations are in progress to make a distinction between the levels of DMP1 and DSPP in sclerotic dentin and their levels in non-affected dentin. The increase in DMP1 levels (37\%) was considerably lower than the increase in DSPP levels (73\%). This discrepancy may be explained speculating that the distinct roles these two proteins (and their products) play in the response to caries might at least partially differ from their functions in physiological dentinogenesis. This is further supported by the evidence of differences in the chemical composition of intratubular dentin in respect to intertubular dentin, as showed by Arnold..$^{40}$

More investigations are though needed to confirm this hypothesis. Ultrastructural studies of DMP1 and DSPP in sclerotic dentin, as well as quantitative and qualitative investigations on their active fragments in carious teeth, are in progress.

\section{References}

1. Fisher LW, Torchia DA, Fohr B, Young MF, Fedarko NS. Flexible structures of SIBLING proteins, bone sialoprotein, and osteopontin. Biochem Biophys Res Commun 2001;280:460-5.

2. Qin C, Baba 0, Butler WT. Post-translational modifications of sibling proteins and their roles in osteogenesis and dentinogenesis. Crit Rev Oral Biol Med 2004;15:126-36.

3. Orsini G, Ruggeri A, Mazzoni A, Nato F, Falconi M, Putignano A, et al. Immunohistochemical localization of dentin matrix protein 1 in human dentin. Eur J Histochem 2008;52:215-20.

4. Deshpande AS, Fang PA, Zhang X, Jayaraman T, Sfeir C, Beniash E. Primary structure and phosphorylation of dentin matrix protein 1 (DMP1) and dentin phosphophoryn (DPP) uniquely determine their role in biomineralization. Bioma cromolecules 2011;12:2933-45.

5. Goldberg M, Smith AJ. Cells and extracellular matrices of dentin and pulp: a biological basis for repair and tissue engineering. Crit Rev Oral Biol Med 2004;15:13-27.

6. Narayanan K, Srinivas R, Ramachandran A, Hao J, Quinn B, George A. Differen tiation of embryonic mesenchymal cells to odontoblast-like cells by overexpression of dentin matrix protein 1. Proc Natl Acad Sci USA 2001;98:4516-21.

7. Ye L, MacDougall M, Zhang S, Xie Y, Zhang $\mathrm{J}$, Li Z, et al. Deletion of dentin matrix protein-1 leads to a partial failure of maturation of predentin into dentin, hypomineralization, and expanded cavities of pulp and root canal during postnatal tooth development. J Biol Chem 2004;279:191418.

8. Lu Y, Ye L, Yu S, Zhang S, Xie Y, McKee $\mathrm{MD}$, et al. Rescue of odontogenesis in Dmp1-deficient mice by targeted reexpression of DMP1 reveals roles for DMP1 in early odontogenesis and dentin apposition in vivo. Dev Biol 2007;303:191201.

9. Turan S, Aydin C, Bereket A, Akcay T, Güran T, Yaralioglu BA, et al. Identification of a novel dentin matrix protein-1 (DMP1) mutation and dental anomalies in a kindred with autosomal recessive hypophosphatemia. Bone 2010;46:402-9.

10. Qin C, Brunn JC, Cook RG, Orkiszewski RS, Malone JP, Veis A, et al. Evidence for the proteolytic processing of dentin matrix protein 1: identification and characterization of processed fragments and cleavage sites. J Biol Chem 2003;278:34700-8.

11. Huang B, Sun Y, Maciejewska I, Qin D, Peng T, McIntyre B, et al. Distribution of SIBLING proteins in the organic and inorganic phases of rat dentin and bone. Eur $\mathrm{J}$ Oral Sci 2008;116:104-12.

12. Sun Y, Lu Y, Chen L, Gao T, D’Souza R, Feng JQ, et al. DMP1 processing is essential to dentin and jaw formation. J Dent Res 2011;90:619-24.

13. Boskey AL. Biomineralization: an overview. Connec Tissue Res 2003;44 (Suppl.1):5-9.

14. Boskey A, Spevak L, Tan M, Doty SB, Butler WT. Dentin sialoprotein (DSP) has limited effects on in vitro apatite formation and growth. Calcif Tissue Int 2000;67:472-8.

15. Suzuki S, Sreenath T, Haruyama N, Honeycutt C, Terse A, Cho A, et al. Dentin sialoprotein and dentin phosphoprotein have distinct roles in dentin mineralization. Matrix Biol 2009;28:221-9.

16. Prasad M, Butler WT, Qin C. Dentin sialophosphoprotein in biomineralization. Connect Tissue Res 2010;51:404-17.

17. Sreenath T, Thyagarajan T, Hall B, Longenecker G, D'Souza R, Hong S, et al. Dentin sialophosphoprotein knockout mouse teeth display widened predentin zone and develop defective dentin mineralization similar to human dentinogenesis imperfecta type III. J Biol Chem 2003;278: 24874-80.

18. Nanci A, Wazen R, Nishio C, Zalzal SF. Immunocytochemistry of matrix proteins in calcified tissues: functional biochemistry on section. Eur J Histochem 2008;52:201-14.

19. Holappa H, Nieminen P, Tolva L, Lukinmaa PL, Alaluusua S. Splicing site mutations in dentin sialophosphoprotein causing dentinogenesis imperfecta type II. Eur J 
Oral Sci 2006;114:381-4.

20. Kim JW, Nam SH, Jang KT, Lee SH, Kim $\mathrm{CC}$, Hahn SH, et al. A novel splice acceptor mutation in the DSPP gene causing dentinogenesis imperfecta type II. Hum Genet 2004;115:248-54.

21. Dong J, Gu T, Jeffords L, MacDougall M. Dentin phosphoprotein compound mutation in dentin sialophosphoprotein causes dentinogenesis imperfecta type III. Am J Med Genet A 2005;132A:305-9.

22. Feng JQ, Luan X, Wallace J, Jing D, Ohshima T, Kulkarni AB, et al. Genomic organization, chromosomal mapping, and promoter analysis of the mouse dentin sialophosphoprotein (Dspp) gene, which codes for both dentin sialoprotein and dentin phosphoprotein. J Biol Chem 1998;273:9457-64.

23. George A, Srinivasan RSR, Liu K, Veis A. Rat dentin matrix protein 3 is a compound protein of rat dentin sialoprotein and phosphophoryn. Connect Tissue Res 1999; 40:49-57.

24. Ritchie HH, Ritchie DG, Wang LH. Six decades of dentinogenesis research. Historical and prospective views on phosphophoryn and dentin sialoprotein. Eur J Oral Sci 1998;106(Suppl.1):211-20.

25. MacDougall M, Zeichner-David M, Slavkin HC. Production and characterization of antibodies against murine dentine phosphoprotein. Biochem J 1985;1232:493-500.

26. Weinstock M, Leblond CP. Radioautographic visualization of the deposition of a phosphoprotein at the mineralization front in the dentin of the rat incisor. J Cell Biol 1973;56:838-45.

27. Stetler-Stevenson WG, Veis A. Type I collagen shows a specific binding affinity for bovine dentin phosphophoryn. Calcif Tissue Int 1986;38:135-41.

28. Orsini G, Ruggeri A, Mazzoni A, Nato F, Falconi M, Putignano A, et al. Immunohi - stochemical localization of dentin matrix protein 1 in human dentin. Eur $\mathbf{J}$ Histochem 2008;52:215-20.

29. Ruggeri A, Orsini G, Mazzoni A, Nato F, Papa V, Piccirilli M, et al. Immunohistochemical and biochemical assay of versican in human sound predentine/dentine matrix. Eur J Histochem 2009;23;53:125-33.

30. Orsini G, Ruggeri A Jr, Mazzoni A, Papa V, Mazzotti G, Di Lenarda R, et al. Immunohistochemical identification of decorin and biglycan in human dentin: a correlative field emission scanning electron microscopy/transmission electron microscopy study. Calcif Tissue Int 2007;81:39-45.

31. Breschi L, Lopes M, Gobbi P, Mazzotti G, Falconi M, Perdigão J. Dentin proteoglycans: an immunocytochemical FEISEM study. J Biomed Mater Res 2002;61:40-6.

32. Mazzoni A, Mannello F, Tay FR, Tonti GA, Papa S, Mazzotti G, et al. Zymographic analysis and characterization of MMP-2 and -9 forms in human sound dentin. $\mathrm{J}$ Dent Res 2007;86:436-40.

33. Weber DF. Human dentine sclerosis: a microradiographic survey. Arch Oral Biol 1974;19:163-9.

34. Blake GC. The peritubular zones in human dentin. Br Dent J 1958;104:57-64.

35. Arnold WH, Konopka S, Kriwalsky MS, Gaengler P. Morphological analysis and chemical content of natural dentin carious lesion zones. Ann Anat 2003;185:419-24.

36. Vahl J, Höhling HJ, Frank RM. Elektronen strahlbeugung an rhomboedrisch aussehenden Mineralbildungen in kariösem Dentin. Arch Oral Biol 1969;9:315-20.

37. Daculsi G, LeGeros RZ, Jean A, Kerebel B. Possible physico-chemical processes in human dentin caries. J Dent Res 1987;66: 1356-9.

38. Riccio M, Resca E, Maraldi T, Pisciotta A, Ferrari A, Bruzzesi G. et al. Human dental pulp stem cells produce mineralized matrix in 2D and 3D cultures. Eur $\mathrm{J}$ Histochem 2010;54:e46.

39. Schroeder HE. [Orale Strukturbiologie]. [Book in German]. Thieme Ed., Stuttgart, Germany, 1992.

40. Arnold WH, Konopka S, Gaengler P. Qualitative and quantitative assessment of intratubular dentin formation in human natural carious lesions. Calcif Tissue Int 2001;69:268-73.

41. Teti G, Salvatore V, Ruggeri A, Manzoli L, Gesi M, Orsini G, et al. In vitro reparative dentin: a biochemical and morphological study. Eur J Histochem 2013;57:e23.

42. Tabatabaei FS, Ai J, Jafarzadeh Kashi TS, Khazaei M, Kajbafzadeh AM, Ghanbari Z. Effect of dentine matrix proteins on human endometrial adult stem-like cells: in vitro regeneration of odontoblasts cells. Arch Oral Biol 2013;58:871-9.

43. Li Y, Lü X, Sun X, Bai S, Li S, Shi J. Odontoblast-like cell differentiation and dentin formation induced with TGF- $\beta 1$. Arch Oral Biol 2011:56:1221-9.

44. Brännström M, Lind PO. Pulpal response to early dental caries. J Dent Res 1965;44: 1045-50.

45. Bjørndal L, Darvann T, Thylstrup A. A quantitative light microscopic study of the odontoblast and subodontoblastic reactions to active and arrested enamel caries without cavitation. Caries Res 1998;32:59-69.

46. Pashley DH. The influence of dentin permeability and pulpal blood flow on pulpal solute concentrations. J Endod 1979;5:355-61.

47. Pashley DH. Dynamics of the pulpo-dentin complex. Crit Rev Oral Biol Med 1996;7: 104-33.

48. Yoshiba K, Yoshiba N, Ejiri S, Iwaku M, Ozawa H. Odontoblast processes in human dentin revealed by fluorescence labeling and transmission electron microscopy. Histochem Cell Biol 2002;118:205-12. 